\title{
FEUILLETAGE À SINGULARITÉS DE VARIÉTÉS DE DIMENSION 3 (THÉORÈME DE J. WOOD)
}

\author{
NGÔ VAN QUÊ
}

\section{Introduction}

Ce travail fait suite à un autre papier [1]. Soit $M$ une $\left(\mathscr{C}^{\infty}\right)$ variété de dim 3. Quoique notre résultat, qui est une conséquence d'un théorème de J. Wood [2], se généralise pour toute variété de $\operatorname{dim} 3$, nous supposerons pour simplifier que $M$ est compacte et orientable.

\section{Rappel: Théorème de J. Wood}

Le fibré tangent $T(M)$ est trivial: $T(M) \simeq M \times R^{3}$. Tout champ de vecteurs $X$ est donc par cet isomorphisme une application $\left(\mathscr{C}^{\infty}\right): M \rightarrow R^{3}$. Etant donné $X$ partout non-nul, rappelons que d'après $\mathrm{J}$. Wood, il existe un champ $Y$ dans la classe d'homotopie $\bar{X}$ do $X$ :

$$
\bar{Y}=\bar{X} \in\left[M, R^{3}-\{0\}\right]
$$

tel que $Y$ soit transverse à un feuilletage de $M$. D'une autre façon de dire $[1, \S 3]$, il existe une 1 -forme $\omega$ intégrable sur $M$, telle que $\omega(\mathrm{Y}) \supsetneqq 0$ sur $M$.

De plus, nous faisons la remarque essentielle suivante: Soit $\Omega$ ouvert de $M: \Omega=\bigcup_{1<i<r} \mathscr{U}_{i}$, réunion d'ouverts de coordonnées $\mathscr{U}_{i}$ difféomorphes au disque $D^{3}$ et à fermeture $\mathscr{U}_{i}$ disjointe:

$$
D^{3}=\left\{\left(x_{1}, x_{2}, x_{3}\right) \in \boldsymbol{R}^{3}|| x_{1}|+| x_{2}|+| x_{3} \mid<1\right\} .
$$

Supposons que sur chaque $\mathscr{U}_{i}, X$ est transverse au feuilletage de $\mathscr{U}_{i}$ défini par la 1 -forme $d x_{3}$. Alors, on pourra prendre $Y$ tel que $\left.Y\right|_{\Omega}=\left.X\right|_{\Omega}$ et le feuilletage de $M$, qui est transverse à $Y$, induise sur chaque $\mathscr{U}_{i}$ le feuilletage considéré, défini par $d x_{3}$.

\section{Champ de vecteurs générique}

Soit $X$ un champ de vecteurs générique sur $M$. De façon précise, si $A$ est un zéro de $X$, alors dans un voisinage de coordonnées $\mathscr{U}$ de $A$ dans $M$ :

Received September 28, 1971. 


$$
\mathscr{U} \underset{A \rightarrow 0}{\widetilde{T}} \boldsymbol{R}^{3} \ni x=\left(x_{1}, x_{2}, x_{3}\right)
$$

$\left.X\right|_{\mathscr{U}}$ est de la forme

$$
X=\sum_{1 \leq i, j \leq 3} \alpha_{j}^{i} x_{i} \frac{\partial}{\partial x_{j}}+g(x)
$$

avec a) $g(x)=0\left(\|x\|^{2}\right)$, b) la matrice $\left(\alpha_{j}^{i}\right)$ a toutes ses valeurs propres $A_{i}=$ $a_{i}+\sqrt{-1} b_{i}$ de partie réelle $a_{i} \neq 0$.

Le lemme suivant est immédiat.

Lemme 1. Soit donné un champ de vecteurs $X$ sur $\boldsymbol{R}^{3}$ de la forme (2.1). Alors le système linéaire de coordonnées $\left(x_{1}, x_{2}, x_{3}\right)$ étant convenablement choisi (la matrice $\left(\alpha_{j}^{i}\right)$ sera en particulier de la forme canonique de Jordan), sur tout voisinage assez petit $\Omega$ de 0 dans $\boldsymbol{R}^{3}$, la 1-forme intégrable $\omega$

$$
\omega=d\left(a_{1} x_{1}^{2}+a_{2} x_{2}^{2}+a_{3} x_{3}^{2}\right)
$$

est transverse à $X$.

Rappelons que $\omega$ est transverse à $X$ si $\omega(X) \supsetneqq 0$ sur $\Omega-\{0\}$.

Autrement dit, au voisinage de tout zéro $A$ de $X, X$ est transverse à un feuilletage singulier à singularité générique $A$. Nous disons encore que $A$ est un zéro sphérique ou conique de $X$ respectivement suivant que $A$ est un point de singularité sphérique ou conique du feuilletage singulier transverse défini au voisinage de $A$.

Rappelons aussi que l'indice du zéro $A$ de $X$ est défini comme \pm 1 , dont le signe est celui du det $\left(\alpha_{j}^{i}\right)=a_{1} \cdot a_{2} \cdot a_{3}$.

\section{Théorème principal}

Le champ générique de vecteurs $X$ étant donné, désignons par $A_{1}, \cdots, A_{2 r}$ les zéros de $X$. Nous considèrerons sur $M$ des champs de vecteurs $Y$ vérifiant:

1) il existe des voisinages $\mathscr{U}_{i}$ des $A_{i}$ dans $M: \Omega=\bigcup_{1 \leq i \leq 2 r} \mathscr{U}_{i}$,

$$
\left.Y\right|_{\Omega}=\left.X\right|_{\Omega}
$$

2) $Y$ et $X$ étant alors des champs de vecteurs partout non-nuls sur $M^{\prime}=$ $M-\Omega,\left.Y\right|_{M^{\prime}}$ et $\left.X\right|_{M^{\prime}}$ sont de même classe d'homotopie, modulo le bord, dans $\left[\left(M^{\prime}, \partial M^{\prime}\right), R^{3}-\{0\}\right]$.

Dans la suite, nous disons simplement qu'un tel champ de vecteurs $Y$ est "homotope" à $X$.

Théorème. Le nombre s de zéros sphériques de $X$ étant inférieur ou égal au nombre $c$ des zéros coniques de $X$, il existe un champ de vecteurs $Y$, homotope à $X$, qui est transverse à un feuilletage à singularités génériques dans $M$. 
Remarque. 1) De façon précise, le feuilletage transverse est défini par une 1-forme intégrable $\omega$ telle que

$$
\omega(Y) \supsetneqq 0 \quad \text { sur } \quad M-\left\{A_{1}, \cdots, A_{2 r}\right\} .
$$

2) L'hypothèse que $s \leq c$ s'introduit naturellement en vue de [1]. A l'aide du théorème de stabilité globale de Reeb, on pourrait montrer que le théorème considéré est faux dans le cas où $s=c+2$.

\section{Démonstration du théorème}

i) Soit $X$ un champ de vecteurs partout non-nuls dans l'espace Euclidien $\boldsymbol{R}^{3}$. Considérons $\gamma:[0,1] \rightarrow \boldsymbol{R}^{3}$ une courbe joignant deux points quelconques $A$ et $B$ de $\boldsymbol{R}^{3}$ tels que

1) $\frac{d \gamma}{d t}(0)=X(A)$ et $\quad \frac{d \gamma}{d t}(1)=X(B)$

2) le produit scalaire Euclidien $\left\langle\frac{d \gamma}{d t}, X[\gamma(t)]\right\rangle \supsetneqq-\left\|\frac{d \gamma}{d t}\right\| \cdot\|X[\gamma(t)]\|$.

$A$ et $B$ étant donnés, l'existence de telles courbes $\gamma$ est facile à établir.

Désignons par $\Omega$ un tronc fermé de cylindre d'âme $\gamma$ :

$$
\Omega=\left\{M \in R^{3} \mid d(M, \gamma)=d(M, \gamma(t)) \leq \rho\right\},
$$

i.e., ensemble de points $M$ dont la distance à $\gamma$ est réalisée par un point $\gamma(t)$ de $\gamma$ et est plus petite qu'un nombre $\rho$ assez petit donné.

Lemme 2. Il existe un champ de vecteurs $Y$ dans $\boldsymbol{R}^{3}$ tel que

1) $\left.Y\right|_{R^{3-} \Omega}=\left.X\right|_{R^{3-\Omega}}$,

2) sur $\Omega, Y$ et $X$ sont de même classe d'homotopie, modulo le bord, dans $\left[(\Omega, \partial \Omega), \boldsymbol{R}^{3}-\{0\}\right]$,

3) $r$ est une trajectoire de $Y$.

Preuve. On peut supposer que $\gamma$ est le segment

$$
\gamma:[0,1] \rightarrow \boldsymbol{R}^{3}, \quad \gamma(t)=(t, 0,0) \in \boldsymbol{R}^{3}
$$

porté par le vecteur unitaire $\vec{u}: \vec{u}=d \gamma / d t$.

Choisissons $\Omega$ de distance $\rho$ assez petite telle que $\forall M \in \Omega,\langle X(M), \vec{u}\rangle \supsetneqq$ $-\|X(M)\|$. Pour $M$ dans $\Omega$, posons $\theta(M), 0 \leq \theta(M)<\pi$, tel que

$$
\langle X(M), \vec{u}\rangle=\|X(M)\| \cos \theta(M),
$$

et désignons par $x(M)$ le point de $\gamma$ réalisant la distance:

$$
d(M, \gamma)=d(M, x(M))
$$

Soit d'autre part $\varphi(x), x \in[0,1]$, une fonction positive, plus petite que $\rho$, et 
ne s'annulant qu'en 0,1 , où elle est plate. Ceci dit, il suffit de prendre pour la famille d'homotopie de champ de vecteurs $Y_{t}, 0 \leq t \leq 1$, telle que

1) $Y_{t}(M)=X(M)$ pour $M$ en dehors de $\Omega$, ou dans $\Omega$ mais $d(M, x(M))$ $\geq \varphi(x(M))$,

2) autrement,

$$
Y_{t}(M)=\operatorname{Rot} .(\vec{v}(M), \quad t \cdot(1-d(M, x(M)) / \varphi(x(M)) \theta(M))[X(M)],
$$

où le second membre est le transformé de $X(M)$ dans la rotation d'angle $t(1-d(M, x(M)) / \varphi[x(M)]) \theta(M)$, d'axe $\vec{v}(M)$, le vecteur unitaire, orthogonal au plan $(X(M), \vec{u})$ et tel que $(\vec{v}(M), X(M), \vec{u})$ forme une base d'orientation positive choisie de $\boldsymbol{R}^{3}$.

ii) Par hypothèse $s \leq c$, nous pouvons associer de façon univoque à tout zéro sphérique $A$ de $X$ un zéro conique $B$ d'indice opposé. Or soient $A$, un zéro sphérique d'indice par exemple +1 , et $B$ le zéro conjque correspondant d'indice -1 . Par le lemme 2, modulo la classe d'homotopie considérée dans $C$, nous pouvons supposer que $X$ admette une trajectoire issue de $A$ et convergeant vers $B$. Ce qui nous donne dans un ouvert de coordonnées contenant $A, B$ et cette trajectoire, une situation semblable à celle du champ de vecteurs suivants dans $\boldsymbol{R}^{3}$ :

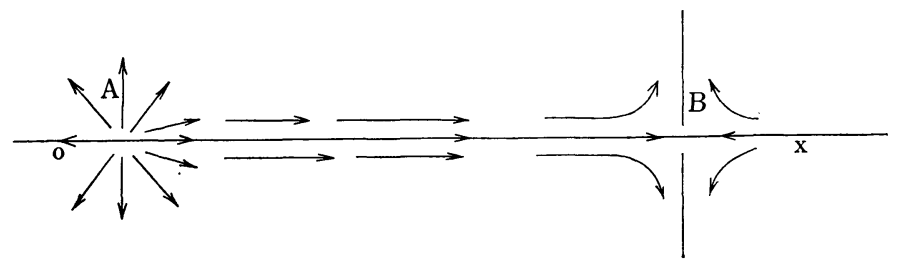

(avec rotation autour de l'axe ox)

Le champ vecteurs $X$ est donc évidemment par le lemme 1 transverse dans un voisinage $\mathscr{U}$, contenant $A$ et $B$, au feuilletage à singularité sphérique $A$ et conique $B$ tel que celui dans $\boldsymbol{R}^{3}$ défini par rotation autour de l'axe ox de la figure suivante:

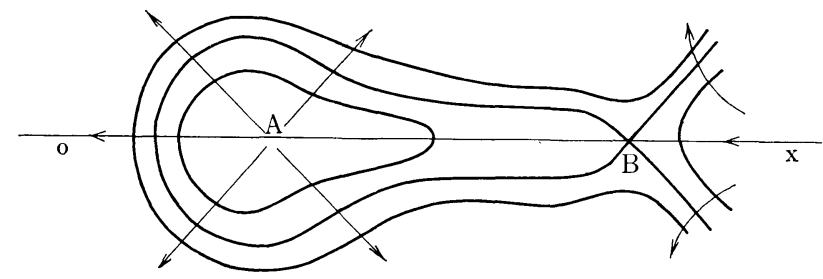

Autrement dit $\mathscr{U}$ étant difféomorphe à

$$
D^{3}=\left\{\left(x_{1}, x_{2}, x_{3}\right) \in \boldsymbol{R}^{3}|| x_{1}|+| x_{2}|+| x_{3} \mid<1\right\} .
$$


$X$ se comporte comme un champ de vecteurs qui est transverse dans $\mathscr{U}$ au feuilletage défini par $d x_{3}$, auquel on a fait une perturbation [1]:

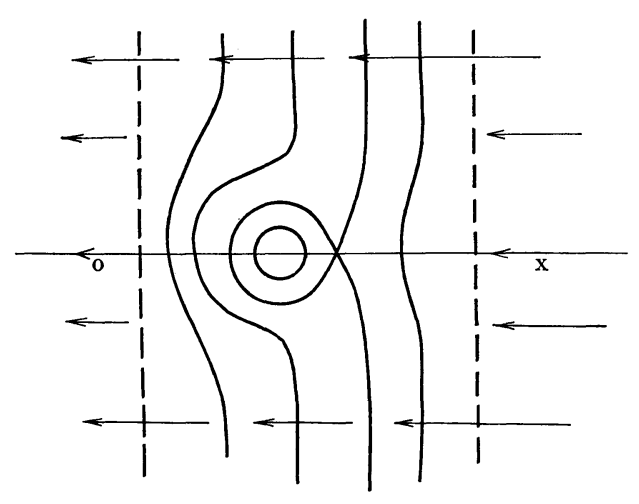

iii) Si $s \leq c-2$, nous groupons encore deux à deux les zéros coniques non considérés de $X$ en couple $(A, B)$ d'indice opposé. Soit donc $A$ un zéro conique d'indice 1 . Modulo la classe d'homotopie considérée dans $C$, il est facile de voir comme conséquence du lemme 1 qu'on peut supposer $X$ être transverse au voisinage de $A$ à un feuilletage à singularité du disque fermé $D^{3}$ (dont le bord est une feuille régulière):

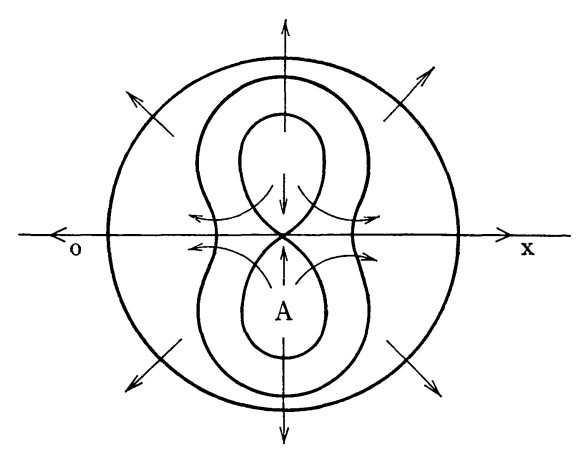

rotation autour de ox, avec une composante de Reeb dans le tore ouvert dont la frontière est la surface engendrée par la courbe en huit. De nouveau, par le lemme 2 , on peut supposer que le point conique $B$ associé d'indice -1 est le point de convergence d'une trajectoire, issue de $A$, de $X$. Et nous sommes comme dans ii): $X$ se comporte dans un voisinage de coordonnées $\mathscr{U}$ contenant $A, B$ et cette trajectoire comme être transverse au feuilletage défini par $d x_{3}$, dans 


$$
\mathscr{U} \simeq\left\{\left(x_{1}, x_{2}, x_{3}\right) \in \boldsymbol{R}^{3}|| x_{1}|+| x_{2}|+| x_{3} \mid<1\right\}
$$

auquel on a fait une perturbation avec deux points coniques:

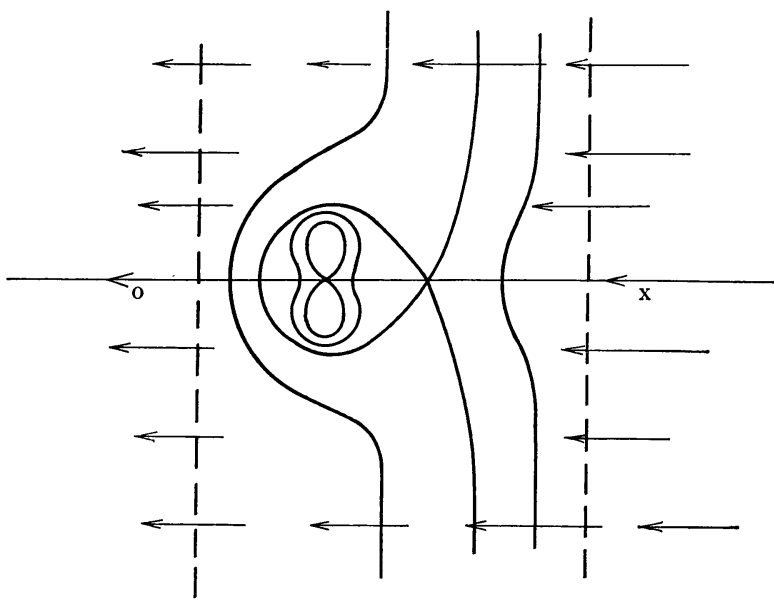

Le théorème annoncé, d'après ce qui précède, n'est plus qu'une conséquence immédiate du théorème de $\mathrm{J}$. Wood et de la remarque que nous avons faite dans $\S 1$.

\section{References}

[1] N. van Quê \& E. Wagneur, Foliations with singularities of 3-manifolds, à paraître. [2] J. W. Wood, Foliations on 3-manifolds, Ann. of Math. 89 (1969) 336-358.

UNIVERSITÉ DE MONTRÉAL 